\section{CLINICALAND EXPERIMENTAL} VACCINE RESEARCH

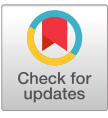

Clin Exp Vaccine Res 2019;8:27-34 https://doi.org/10.7774/cevr.2019.8.1.27 pISSN 2287-3651 • elSSN 2287-366X

Satish S. Gaikwad', Hyun-Jeong Lee², Ji-Ye Kim ${ }^{3}$, Kang-Seuk Choi ${ }^{4}$

'Department of Veterinary Microbiology, College of Veterinary and Animal Sciences, Parbhani, India; ${ }^{2}$ Avian Disease Research Division, ${ }^{3}$ Veterinary Drugs and Biologics Division, and ${ }^{4}$ Planning and Coordination Division, Animal and Plant Quarantine Agency, Gimcheon, Korea

Received: November 22, 2018

Revised: January 1, 2019

Accepted: January 7, 2019

Corresponding author: Kang-Seuk Choi, DVM, PhD Planning and Coordination Division, Animal and Plant Quarantine Agency, 177 Hyeokshin 8-ro, Gimcheon 39660, Korea

Tel: +82-54-912-0341, Fax: +82-54-912-0338

E-mail:kchoi0608@korea.kr

No potential conflict of interest relevant to this article was reported.

This work was supported by the Animal and Plant Quarantine Agency, Korea (grant no. B-15430842014-15-02).

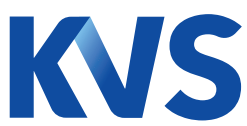

KOR E A N

VACCIN E

SO CI E T Y

(C) Korean Vaccine Society.

This is an Open Access article distributed under the terms of the Creative Commons Attribution Non-Commercial License (http://creativecommons.org/licenses/ by-nc/4.0) which permits unrestricted non-commercial use, distribution, and reproduction in any medium, provided the original work is properly cited.
Expression and serological application of recombinant epitope-repeat protein carrying an immunodominant epitope of Newcastle disease virus nucleoprotein

Purpose: The aim of the present study was to develop a serodiagnostic test for differentiation infected from vaccinated animal (DIVA) strategy accompanying the marker vaccine lacking an immunodominant epitope (IDE) of nucleoprotein of Newcastle disease virus (NDV).

Materials and Methods: Recombinant epitope-repeat protein (rERP) gene encoding eight repeats of the IDE sequence (ETQFLDLMRAVANSMR) by tetra-glycine linker was synthesized. Recombinant baculovirus carrying the rERP gene was generated to express the rERP in insect cells. Specificity and sensitivity of an indirect enzyme-linked immunosorbent assay (ELISA) employing the rERP was evaluated.

Results: The rERP with molecular weight of $20 \mathrm{kD}$ a was successfully expressed by the recombinant baculovirus in an insect-baculovirus system. The rERP was antigenically functional as demonstrated by Western blotting. An indirect ELISA employing the rERP was developed and its specificity and sensitivity was determined. The ELISA test allowed discrimination of NDV infected sera from epitope deletion virus vaccinated sera.

Conclusion: The preliminary results represent rERP ELISA as a promising DIVA diagnostic tool.

Keywords: Newcastle disease virus, Nucleoprotein, Epitope-repeat protein, Enzyme-linked immunosorbent assay, Differentiation infected from vaccinated animal

\section{Introduction}

Newcastle disease virus (NDV) is the etiological agent of Newcastle disease (ND), one of the most infectious diseases affecting poultry. The virus has an enveloped non-segmented negative stranded RNA genome and is member of the genus Avulavirus in the family Paramyxoviridae. ND outbreaks cause mortality rate up to $100 \%$ in susceptible poultry and is a notifiable disease to the World Organisation for Animal Health (OIE) [1]. The NDV genome consists of 55 nt leader at its $3^{\prime}$ end and $114 \mathrm{nt}$ trailer at its $5^{\prime}$ end, flanking six essential genes encoding nucleoprotein (NP), matrix protein (M), phosphoprotein $(\mathrm{P})$, fusion protein $(\mathrm{F})$, hemagglutinin-neuraminidase protein $(\mathrm{HN})$, and large polymerase protein $(\mathrm{L})$ [2].

Internal structural protein NP is a highly conserved protein involved in virus replication cycle as well as in inducing high level of NDV-specific antibody in chickens [3]. 
$\mathrm{N}$ - and C-terminal regions of the NP serve as B-cell epitopes in host $[4,5]$. In particular, C-terminal immunological region that forms alpha helical structure is permissible for modification. Thus this epitopic region can serve as negative marker if replaced with a foreign epitope [6]. For example, amino acid sequence ETQFLDLMRAVANSMR (aa 444-459) on NDV NP is an epitope, which serves as linear immunodominant epitope (IDE) and can be replaced or deleted [4]. This antigen should facilitate differentiation of vaccinated animal to that of infected during serosurveillance in the case of the marker vaccine applying vaccination program. A multiepitope protein as a vaccine candidate [7-12] or as diagnostic reagent [13-15] has been explored in multiple instances. Multiepitope recombinant proteins are advantageous as they can be designed rationally and offers higher specificity and sensitivity [16].

The aim of the present study was to develop a serodiagnostic test for differentiation infected from vaccinated animal (DIVA) strategy accompanying an NDV marker vaccine lacking the NP IDE. For this purpose, an indirect enzyme-linked immunosorbent assay (ELISA) format employing a recombinant protein, which carries multimers of IDE, was developed. The recombinant protein was expressed in baculovirus system. Specificity and sensitivity of recombinant protein was evaluated.

\section{Materials and Methods}

\section{Design of synthetic gene and tertiary structure prediction}

Epitope repeat protein (ERP) gene encoding eight repeats of an IDE sequence containing ETQFLDLMRAVANSMR (C-terminal IDE aa 444-459) on NDV NP separated by tetra-glycine linker was synthesized commercially using codon optimization for baculovirus expression. The recognition enzyme sites of EcoRI and HindIII were built in the upstream and downstream of coding sequence, respectively. The synthetic gene cloned in vector backbone PMK-RQ (KanR) was received as $5 \mu \mathrm{g}$ of lyophilized plasmid preparation. The conceptual translated sequence was submitted to I-TASSER server for homology modeling [17] and top five predicted structures were visualized with PyMOL software [18].

\section{Preparation of recombinant bacmid}

Synthetic gene was subcloned into pFastBac HT b vector using EcoRI and HindIII restriction sites. The recombinant plasmid (50 ng) was transformed in Escherichia coli DH B10 cells.
Transformed cells were plated onto the Luria-Bertani agar containing kanamycin $(50 \mu \mathrm{g} / \mathrm{mL})$, gentamicin $(7 \mu \mathrm{g} / \mathrm{mL})$, tetracycline $(10 \mu \mathrm{g} / \mathrm{mL})$, Bluo-gal $(100 \mu \mathrm{g} / \mathrm{mL})$, and isopropylthio- $\beta$-galactoside (IPTG, $40 \mu \mathrm{g} / \mathrm{mL}$ ) and incubated at $37^{\circ} \mathrm{C}$ for 36 to 48 hours. The high molecular weight bacmid DNA was isolated from the overnight cultures by alkaline lysis purification according to the manufacturer's manual of Bac-toBac baculovirus expression system (Invitrogen, Carlsbad, CA, USA).

\section{Generation of recombinant baculovirus}

Spodoptera frugiperda 9 (Sf9) cells were cultured at $27^{\circ} \mathrm{C}$ in Sf-900 II serum free medium (Invitrogen), supplemented with $10 \%$ heat-inactivated fetal bovine serum (FBS) (Invitrogen), $50 \mathrm{U} / \mathrm{mL}$ penicillin and $50 \mu \mathrm{g} / \mathrm{mL}$ streptomycin. Sf9 cells were transfected with recombinant bacmid DNA using Cellfectin II, a cationic lipid for the transfection of the baculovirus particles according to the manufacturer's instructions. Briefly, for each transfection, $2 \mathrm{~mL}$ of Grace's Insect Medium, unsupplemented (without antibiotics and serum) was added in each well, $8 \times 10^{5}$ cells per well were seeded in a 6 -well plate and allowed to attach for 2 hours. The bacmid DNA and Cellfectin II ( $8 \mu \mathrm{L}$ of reagent) were diluted separately in $100 \mu \mathrm{L}$ of Grace's medium, unsupplemented (without antibiotics and serum), then mixed and incubated for 30 minutes at room temperature to form lipid-DNA complexes. The cells were washed with fresh medium, and incubated with lipid-DNA complex at $27^{\circ} \mathrm{C}$ for 5 hours. The transfection solution was removed and $2 \mathrm{~mL}$ supplemented Sf-900 II SFM containing $10 \%$ FBS was added. Transfected Sf9 cells were incubated at $27^{\circ} \mathrm{C}$ for 72 hours for baculovirus production. Recombinant baculovirus production was monitored daily by visualization of the cytopathic effects. Three to four days after transfection, recombinant baculovirus was harvested from the cell culture medium and stored at $4^{\circ} \mathrm{C}$. Recombinant viruses were identified by polymerase chain reaction using gene vector specific primers (Invitrogen). The resulting baculovirus was passaged three times by infecting more Sf9 cells.

\section{Protein expression and purification}

SF9 suspension cultures $\left(2 \times 10^{6}\right.$ cells $\left./ \mathrm{mL}\right)$ grown in $2 \mathrm{~L}$ of Sf900 II medium supplemented with $1 \%$ of FBS were infected with recombinant baculovirus at multiplicity of infection of 5 plaque-forming unit/cell. Cells were grown for 72 hours and cell pellets were washed in phosphate buffered saline (PBS). As recombinant ERP (rERP) carried $6 \times$ His tag at N-terminal, 
it was purified by using Ni-NTA spin columns under denaturant condition with $8 \mathrm{M}$ urea according to Ni-NTA spin handbook (Qiagen, Hilden, Germany). The collected cells were lysed in $10 \mathrm{~mL}$ buffer $\mathrm{B}\left(10 \mathrm{mM} \mathrm{NaH}_{2} \mathrm{PO}_{4}, 300 \mathrm{mM} \mathrm{NaCl}, 8 \mathrm{M}\right.$ urea, $\mathrm{pH}$ 8.0) and centrifuged the lysate at $10,000 \times \mathrm{g}$ for 30 minutes and collecting supernatant. Loading up to $600 \mu \mathrm{L}$ of the cleared lysate supernatant containing the $6 \times$ His-tagged protein onto a pre-equilibrated Ni-NTA spin column, centrifuged 2 minutes at $700 \times \mathrm{g}$ and washing the column with 600 $\mu \mathrm{L}$ buffer C $\left(10 \mathrm{mM} \mathrm{NaH}_{2} \mathrm{PO}_{4}, 300 \mathrm{mM} \mathrm{NaCl}, 8 \mathrm{M}\right.$ urea, $\mathrm{pH}$ 6.3) and centrifuged 2 minutes at $700 \times \mathrm{g}$, the recombinant protein was finally eluted with buffer $\mathrm{E}\left(10 \mathrm{mM} \mathrm{NaH}_{2} \mathrm{PO}_{4}, 300\right.$ $\mathrm{mM} \mathrm{NaCl}, 8 \mathrm{M}$ urea, $\mathrm{pH}$ 4.3). The recombinant protein was allowed to refold using refolding buffer (50 mM Tris $\mathrm{pH} 7.5$, $0.5 \mathrm{M} \mathrm{NaCl}, 0.3 \%$ CHAPS, 1 mM DTT, 5\% glycerol). rERP was dialyzed against refolding buffer (40 mM CHAPS and $10 \mathrm{mM}$ DTT).

\section{Electrophoresis and Western blotting}

Proteins extracted from infected Sf9 cells were fractionated by $10 \%$ sodium dodecyl sulfate polyacrylamide gel electrophoresis under reducing conditions. The separated proteins were blotted onto an Immun-Blot polyvinylidene fluoride membrane (Bio-Rad, Richmond, CA, USA) using a wet transfer system (Bio-Rad). The membrane was blocked with 5\% skim milk in PBS containing 0.1\% Tween 20 (PBST) at room temperature for 1 hour. After washing 3 times with PBST, the blocked membrane was subsequently incubated with NDV chicken antiserum (diluted 1:100) for 1 hour, rinsed in PBST, and incubated with alkaline phosphatase-conjugated goat anti-chicken immunoglobulin (diluted 1:1,000, Pierce, Rockford, IL, USA) for 1 hour. Protein bands were visualized by enhanced chemiluminescence using NBT/BCIP solution (Sigma-Aldrich, St. Louis, MO, USA).

\section{Standardization of the indirect ELISA}

Optimal dilutions of rERP and sera were determined by a checkerboard titration test with NDV positive and negative sera previously confirmed by hemagglutionation inhibition (HI) test. The antigen was coated in 96-well ELISA plates (PolySorb, Nunc, Roskilde, Denmark) ranging in concentration from 2 $\mu \mathrm{g} / \mathrm{mL}$ to $0.0625 \mu \mathrm{g} / \mathrm{mL}$ in $50 \mathrm{mM}$ carbonate/bicarbonate buffer (pH 9.6). Reference positive and negative sera were both diluted serially from 1:100-1:6,400 and tested to determine the optimal serum dilution. The dilutions that gave the optimum difference in absorbance at $450 \mathrm{~nm}$ between posi- tive and negative sera were selected to test the sera panel. The working dilution of goat anti-chicken HRP-IgG (Sigma-Aldrich), the reaction temperature, time and other conditions also were optimized.

Ten NDV negative serum samples from specific pathogen free (SPF) chickens were used to determine a cutoff value for the ELISA assay. The mean negative serum optical density (OD) value plus three standard deviations (SD) was used as the cutoff value. Each sample was repeated in triplicate wells and the mean value was calculated. The experiment was repeated two more times. In addition to SPF chicken serum, different hyper-immune chicken sera to NDV, H9N2 avian influenza virus (AIV), infectious bronchitis virus (IBV), and infectious bursal disease virus (IBDV) were tested for specificity of rERP using an indirect ELISA assay. To test the sensitivity of the rERP, the chicken anti-NDV serum was diluted serially (1:100-1:6,400) and the serum with each dilution was used to react with the expressed rERP in an indirect ELISA.

\section{rERP ELISA}

A total of 30 chicken sera comprising three groups with each group of 10 were used in the study. These sera were kept at the OIE reference laboratory for Newcastle disease, Animal and Plant Quarantine Agency (APQA), Korea. First group were sera taken from mock-infected SPF chicken (NDV antibody negative). Second and third groups were sera taken $14 \mathrm{dpi}$ from SPF chickens vaccinated with IDE deleted recombinant Newcastle disease virus (rNDV) [4] and NDV LaSota strain [19], respectively, which kindly supplied by the OIE reference laboratory for ND, Korea. All vaccinated sera were proved to be serologically positive by a HI test using NDV antigen. All sera were available with the laboratory. Microtiter plates were coated with $100 \mu \mathrm{L}$ of $1 \mu \mathrm{g} / \mathrm{mL}$ rERP protein in $50 \mathrm{mM}$ carbonate/bicarbonate buffer $\mathrm{pH}$ 9.6, and incubated overnight at $4^{\circ} \mathrm{C}$. Plates were washed three times with PBST and blocked with $150 \mu \mathrm{L}$ per well PBST with 5\% skim milk powder solution at $37^{\circ} \mathrm{C}$ for 2 hours. The chicken anti-NDV serum (1:400) was added to each. After 1-hour incubation at $37^{\circ} \mathrm{C}$, plates were washed three times with PBST. One hundred microliters horseradish peroxidase-conjugated goat anti-chicken IgG (Pierce) at 1:2,000 dilution was added to each well and plates were incubated at $37^{\circ} \mathrm{C}$ for 1 hour. Then plates were washed three times with PBST and $100 \mu \mathrm{L}$ TMB (3,3,5,5-tetramethylbenzidine) was added to each well. After 10-minute incubation at $37^{\circ} \mathrm{C}$, reactions were stopped with $2 \mathrm{M} \mathrm{H}_{2} \mathrm{SO}_{4}$ and the $\mathrm{OD}$ at $450 \mathrm{~nm}$ was measured with microplate reader (Sunrise, 
Satish S. Gaikwad et al • rERP of NDV nucleoprotein

Tekan, Männedorf, Switzerland).

\section{Statistical analysis}

Mean ELISA titer and SDs of each group were calculated using Microsoft Excel (Microsoft, Redmond, WA, USA). Differences in ELISA absorbance between groups were analyzed by the one-way analysis of variance (ANOVA). A p-value of $<0.05$ was considered statistically significant.

\section{Results}

\section{Construction of bacmid and tertiary structure prediction of recombinant protein}

The construct encoding ERP on NDV NP was designed as shown in Fig. 1A. The codon optimized gene (486 bp) was designed to have 8 repeats of 16 amino acids (ETQFLDLMRAVANSMR) mimicking C-terminal IDE (aa 444-459) on NDV NP. rERP gene insert was excised from the plasmid after digestion with EcoRI and HindIII enzymes. Resulting fragment
(486 bp) was subcloned into the pFastBac HT b vector using above mentioned restriction sites. Recombinant baculovirus containing the recombined ERP gene insert (bac-rERP) was generated by transposition of recombinant vector into the SF9 cells by transfection. The codon optimized gene was commercially synthesized from GeneArt (Regensburg, Germany) and subcloned into baculovirus transfer vector. Alignment of obtained nucleotide and deduced amino acid sequences of the rERP gene insert revealed $100 \%$ match with the designed construct (data not shown). I-TASSER server predicted 5 models, all of which showed optimal spacing in epitopes separated by linkers (Fig. 1B). Graphic visualization of tertiary structure of top predicted models for rERP protein suggests that IDE reactive antibodies would be freely accessible to epitopes separated by tetra-glycine linkers in tertiary dimensional space.

\section{Expression, purification, and characterization of rERP}

Bac-to-Bac expression system was used to produce a baculovirus encoding $\mathrm{N}$-terminally $6 \times$ His tagged rERP protein un-
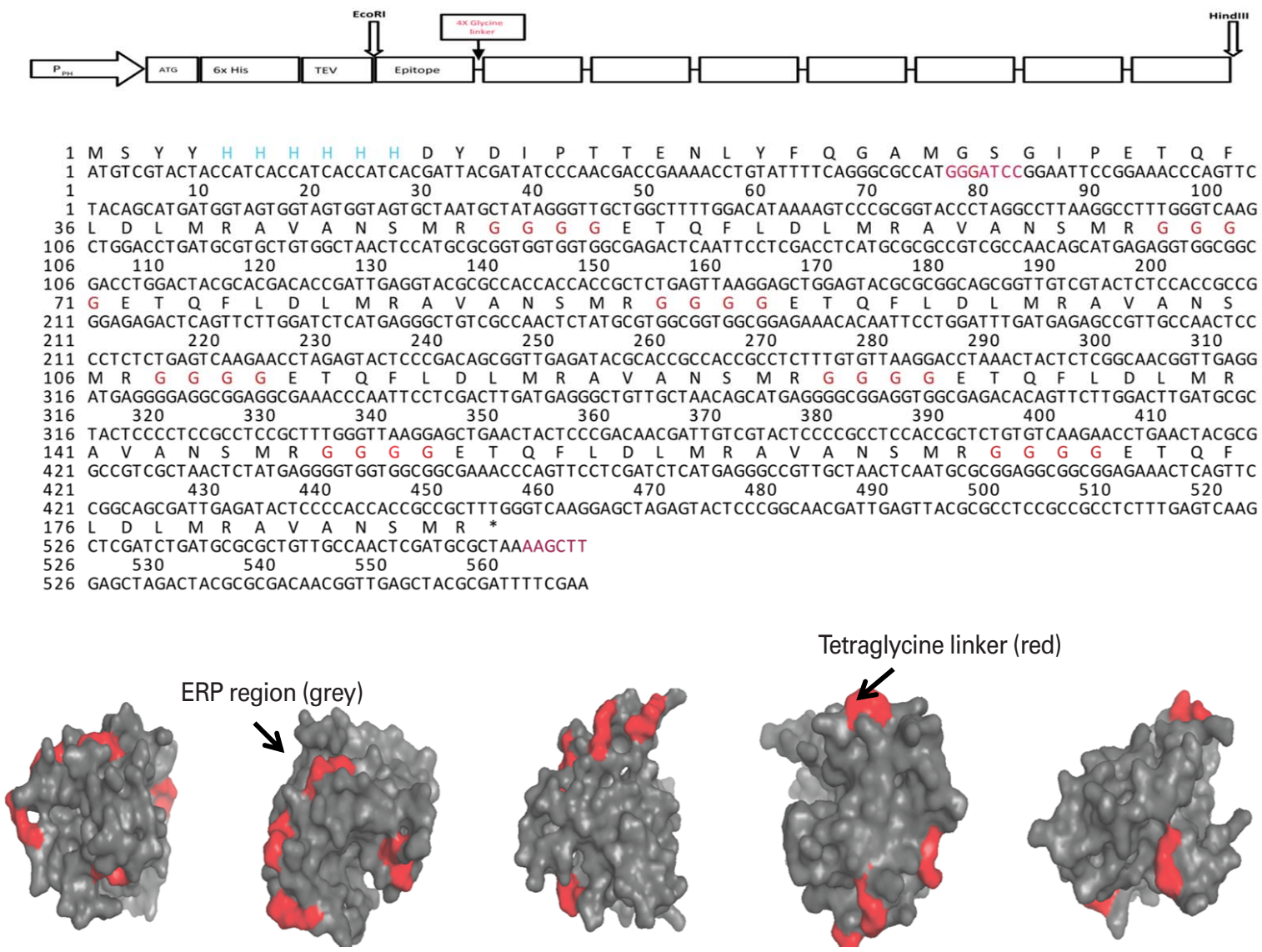

Fig. 1. Design and the complete nucleotide and predicted amino acid sequence of the recombinant epitope-repeat protein (rERP) protein (A). Graphic visualization of tertiary structure of top predicted models for rERP protein with flexible linker generated by I-TASSER server (B): the protein model suggests freely accessible epitopes separated by tetra-glycine linkers in three dimensional space. 
der the transcriptional control of polyhedrin promoter. Recombinant baculovirus bac-rERP infected cells were lysed under denaturing conditions and rERP was purified using His tag purification. The protein was allowed to refold using refolding buffer. To identify the expressed rERP, the purified protein was analyzed by western blot assay. The chicken antiNDV serum raised by infecting SPF chickens with La Sota strain of NDV [19] was used as the primary antibody in the analysis. The results showed purified rERP with molecular weight of approximately $20 \mathrm{kDa}$ reacted specifically (lanes 1 to 6 in Fig. 2) to the NDV positive serum. No specific band was detected with negative controls (lanes N1 and N2 in Fig. 2), which indicated that the rERP is expressed correctly, and has

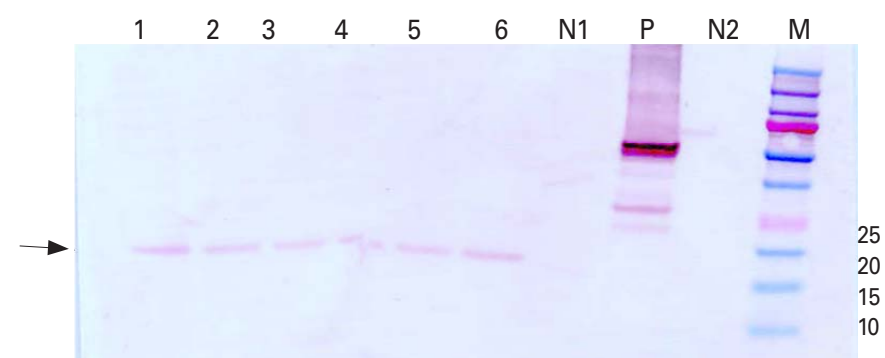

Fig. 2. Identification of the recombinant epitope-repeat protein (rERP). Lanes 1 to 6, rERP protein; lane P, positive control (Newcastle disease virus LaSota); lane N1, negative control (cell culture fluid); lane N2, negative control (phosphate buffered saline); $\mathrm{M}$, protein marker.

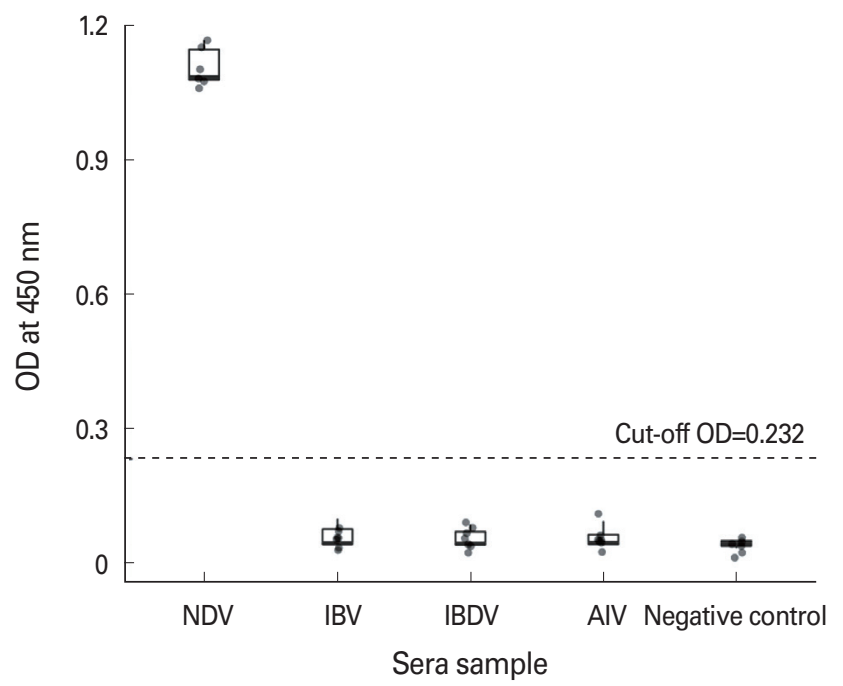

good reaction ability with chicken specific anti-NDV serum.

\section{Specificity and sensitivity of the rERP}

The optimal concentration of the $\mathrm{rERP}(1 \mu \mathrm{g} / \mathrm{mL})$ and the dilution rate of sera (1:400) for indirect ELISA assays to evaluate the specificity and sensitivity of the expresses rERP were determined. To set up a cutoff value for indirect ELISA assay, 10 SPF chicken serum samples were analyzed. The mean $\mathrm{OD}_{450}$ value of these samples as measured by ELISA was 0.231 with a SD of 0.00022 . For a $99 \%$ confidence interval, the cutoff value determined was 0.232 (mean OD of SPF sera samples+3 SD). Based on the cutoff value, the reactivity of rERP with chicken anti-AIV, anti-IBV, anti-IBDV, and normal sera was measured. The result showed that OD450 $\mathrm{nm}$ values of non-NDV sera were lower than cutoff value (Fig. 3A). The minimum dilution titer of chicken anti-NDV serum detected was 1:1,600 according to cutoff value of 0.232 (Fig. 3B).

\section{rERP ELISA}

A battery of 30 chicken sera samples divided in ten chickens per group in three was tested. Samples in groups of mock infected chicken sera and chicken vaccinated with the IDE deleted rNDV showed OD values below cut-off value. The OD value for samples from wildtype NDV LaSota vaccinated chicken group were in the range of 0.255 to 1.77 , which was above determined cut-off value (Fig. 4). The indirect ELISA showed

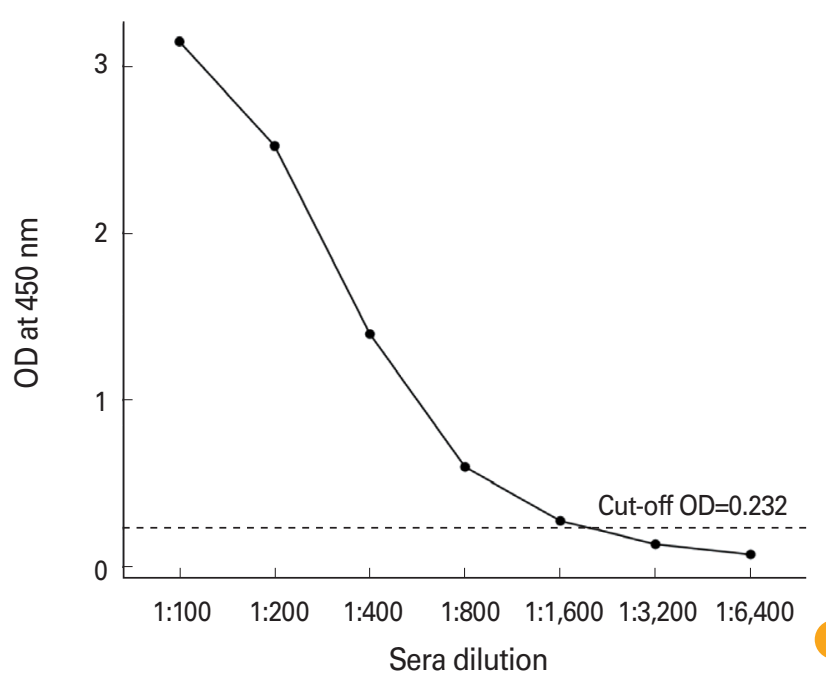

Fig. 3. Specificity and sensitivity of the recombinant epitope-repeat protein (rERP). (A) Specificity of the rERP. Different serum samples of chicken anti-Newcastle disease virus (NDV), anti-infectious bursal disease virus (IBDV), anti-infectious bronchitis virus (IBV), anti-avian influenza virus (AIV), and specific pathogen free serum as negative control were tested by indirect enzyme-linked immunosorbent assay (ELISA). Boxes denote interquartile ranges, with median values shown as horizontal lines inside the box. Whiskers denote ranges. Individual data points are plotted. (B) Sensitivity of the rERP. Serial dilutions of NDV positive serum (from 1:100 to 1:6,400) were tested by an indirect ELISA. The results showed that the minimum detection limit of chicken anti-NDV positive sera was 1:1,600. 


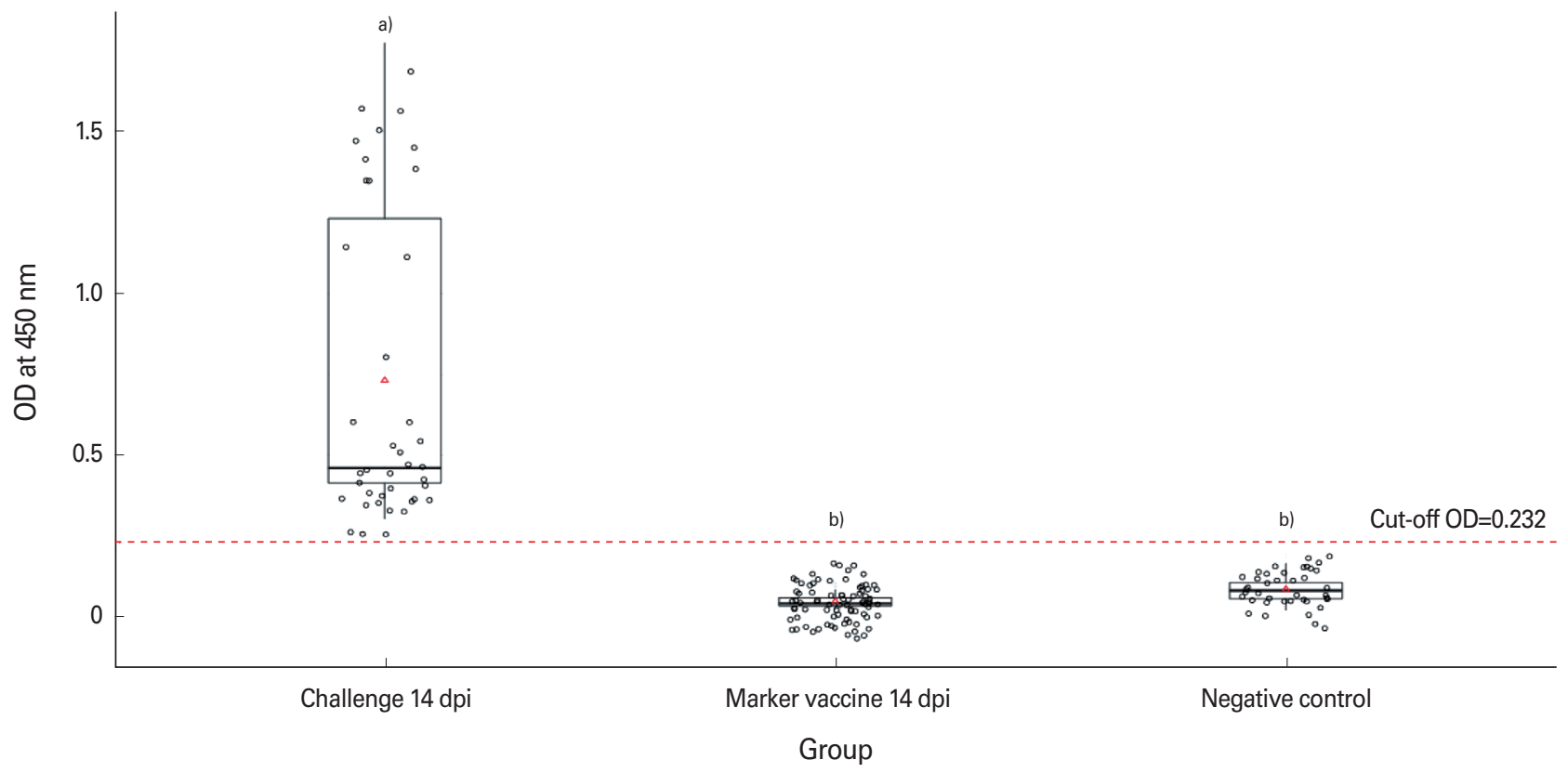

Fig. 4. Box-and-whisker plot of absorbance values of indirect enzyme-linked immunosorbent assay in three different groups. Boxes denote interquartile ranges, with median values shown as horizontal lines inside the box. Whiskers denote ranges. Triangle represents mean values. All data points from 4 independent experiments of sample size $n=10$ in each group are plotted. ${ }^{a \mid, b}$ Superscript carrying different alphabets are significantly different $(p<0.05)$.

good repeatability through the multiple experiments.

\section{Discussion}

Marker NDV vaccines in conjugation with serological diagnostic test has substantial value in poultry industry for demonstration of the freedom of ND. A serologic marker antigen is a viral protein or an epitope that is absent from the vaccine strains but consistently present in the corresponding wildtype viruses [5]. Therefore, only animals that have been infected with wild-type virus will develop antibodies against the serologic marker antigen while the vaccinated animals will not. The marker antigen should be conserved and immunodominant to ensure that the companion diagnostic test based on this marker antigen will produce reliable diagnostic results [20]. In addition, the marker antigen should be dispensable for the viral life cycle but should not contribute significantly to the overall immunogenicity of the vaccine. Subunit vaccines using $\mathrm{F}$ and $\mathrm{HN}$ protein [21] can serve as marker vaccines but are less effective than whole virus vaccines [22]. This necessitates NDV marker vaccine based on chimeric live virus.

NDV NP is known to be carry three antigenic regions, two of which are located at $\mathrm{N}$ terminal [5]. C terminal IDE (aa res- idues 444-459) ETQFLDLMRAVANSMR is a dispensable epitope on NP protein, which can be replaced with foreign epitope [4]. In preliminary study, when chickens were immunized with viable recombinant NDV lacking this IDE with reverse genetics technique, monomeric IDE peptide based ELISA showed poor sensitivity most likely due to small hapten size of 16 aa and immunosteric hindrance to bovine serum albumin conjugated peptide (data not shown). To address this issue, this problem can be solved by using multimers of IDE in the form of recombinant protein antigen.

In the study, we designed, expressed and characterized the rERP in baculovirus expression system. This protein carried tandem repeats of IDE on NDV NP. The protein carried N terminal his tag allowing one-step purification under Ni NTA agar column $[23,24]$. The construct designed to express recombinant protein was codon optimized for Sf9 cells. Codon optimization involves codon adaptability, mRNA structure, tRNA usage. It significantly increases protein expression [2527]. We used tetra-glycine linker as linker in between epitopes as it provides flexibility due to lack of $\beta$-carbon and is preferred linker in multi-epitope proteins [28]. Computer modeling showed that all the epitopes are freely accessible in three-dimensional space. This rERP was expressed at high concentration in insect cells when titrated by ELISA. We purified pro- 
tein under denaturing condition on a Ni-NTA matrix with a high degree of purity [29].

Western blot analysis showed the purified rERP was recognized by chicken anti-NDV serum with a specific band at approximately $20 \mathrm{kDa}$ (lanes 1-6 in Fig. 2), while no specific band was observed when using negative control serum. This indicates that the expressed rERP has the molecular weight as expected. We employed the indirect ELISA to evaluate the specificity and sensitivity of the expressed rERP using confirmed positive and negative sera samples. rERP could react specifically with the anti-NDV serum and showed no reactivity towards anti-IBV, anti-IBDV, anti-AIV, and normal sera. It suggests that rERP can specifically recognize anti-NDV antibodies. The potential of rERP ELISA to serve as discriminatory test in combination with the IDE deleted marker vaccine was investigated using three panels of sera. The test correctly classified the marker vaccinated and SPF chicken sera as antibody negative but sera from wildtype NDV immunized chickens as antibody positive. These results revealed that rERP possessed good reactivity, specificity and sensitivity.

Future work involves testing sera samples under field trials. If proven satisfactory, the recombinant protein represents a promising candidate as companion diagnostic test in NDV DIVA vaccination program. This strategy of epitope-based recombinant proteins as ELISA antigens against modification permissible regions of viruses makes it a highly effective approach in DIVA vaccination.

\section{ORCID}

Satish S. Gaikwad https://orcid.org/0000-0002-1031-5677

Hyun-Jeong Lee https://orcid.org/0000-0002-0983-3696

Ji-Ye Kim https://orcid.org/0000-0001-8441-8903

Kang-Seuk Choi https://orcid.org/0000-0001-6825-6924

\section{References}

1. Miller PJ, Torchetti MK. Newcastle disease virus detection and differentiation from avian influenza. Methods Mol Biol 2014;1161:235-9.

2. Diel DG, da Silva LH, Liu H, Wang Z, Miller PJ, Afonso CL. Genetic diversity of avian paramyxovirus type 1: proposal for a unified nomenclature and classification system of Newcastle disease virus genotypes. Infect Genet Evol 2012; 12:1770-9.

3. Mohan CM, Dey S, Rai A, Kataria JM. Recombinant hae- magglutinin neuraminidase antigen-based single serum dilution ELISA for rapid serological profiling of Newcastle disease virus. J Virol Methods 2006;138:117-22.

4. Mebatsion T, Koolen MJ, de Vaan LT, et al. Newcastle disease virus (NDV) marker vaccine: an immunodominant epitope on the nucleoprotein gene of NDV can be deleted or replaced by a foreign epitope. J Virol 2002;76:10138-46.

5. Ahmad-Raus R, Ali AM, Tan WS, Salleh HM, Eshaghi M, Yusoff K. Localization of the antigenic sites of Newcastle disease virus nucleocapsid using a panel of monoclonal antibodies. Res Vet Sci 2009;86:174-82.

6. Uttenthal A, Parida S, Rasmussen TB, Paton DJ, Haas B, Dundon WG. Strategies for differentiating infection in vaccinated animals (DIVA) for foot-and-mouth disease, classical swine fever and avian influenza. Expert Rev Vaccines 2010;9:73-87.

7. AnandaRao R, Swaminathan S, Fernando S, Jana AM, Khanna N. A custom-designed recombinant multiepitope protein as a dengue diagnostic reagent. Protein Expr Purif 2005;41:136-47.

8. Huang XJ, Lu X, Lei YF, et al. Cellular immunogenicity of a multi-epitope peptide vaccine candidate based on hepatitis C virus NS5A, NS4B and core proteins in HHD-2 mice. J Virol Methods 2013;189:47-52.

9. Oany AR, Emran AA, Jyoti TP. Design of an epitope-based peptide vaccine against spike protein of human coronavirus: an in silico approach. Drug Des Devel Ther 2014;8: 1139-49.

10. Spearman P, Kalams S, Elizaga M, et al. Safety and immunogenicity of a CTL multiepitope peptide vaccine for HIV with or without GM-CSF in a phase I trial. Vaccine 2009; 27:243-9.

11. Cho HI, Celis E. Design of immunogenic and effective multiepitope DNA vaccines for melanoma. Cancer Immunol Immunother 2012;61:343-51.

12. Tian L, Wang HN, Lu D, Zhang YF, Wang T, Kang RM. The immunoreactivity of a chimeric multi-epitope DNA vaccine against IBV in chickens. Biochem Biophys Res Commun 2008;377:221-5.

13. de Souza MQ, Galdino AS, dos Santos JC, et al. A recombinant multiepitope protein for hepatitis B diagnosis. Biomed Res Int 2013;2013:148317.

14. Bhatnagar S, Kumar P, Mohan T, et al. Evaluation of multiple antigenic peptides based on the Chikungunya E2 protein for improved serological diagnosis of infection. Viral Immunol 2015;28:107-12. 
15. Cheng Z, Zhao JW, Sun ZQ, et al. Evaluation of a novel fusion protein antigen for rapid serodiagnosis of tuberculosis. J Clin Lab Anal 2011;25:344-9.

16. Lin X, Chen Y, Yan J. Recombinant multiepitope protein for diagnosis of leptospirosis. Clin Vaccine Immunol 2008; 15:1711-4.

17. Zhang Y. I-TASSER: fully automated protein structure prediction in CASP8. Proteins 2009;77 Suppl 9:100-13.

18. DeLano WL. The PyMOL molecular graphics system. Palo Alto: DeLano Scientific; 2002.

19. Jeon WJ, Lee EK, Lee YJ, et al. Protective efficacy of commercial inactivated Newcastle disease virus vaccines in chickens against a recent Korean epizootic strain. J Vet Sci 2008;9:295-300.

20. Chander V, Nandi S, Ravishankar C, Upmanyu V, Verma R. Classical swine fever in pigs: recent developments and future perspectives. Anim Health Res Rev 2014;15:87-101.

21. Takada A, Kida H. Protective immune response of chickens against Newcastle disease, induced by the intranasal vaccination with inactivated virus. Vet Microbiol 1996;50: 17-25.

22. Kamiya N, Niikura M, Ono M, Kai C, Matsuura Y, Mikami T. Protective effect of individual glycoproteins of Newcastle disease virus expressed in insect cells: the fusion protein derived from an avirulent strain had lower protective efficacy. Virus Res 1994;32:373-9.
23. Guan D, Chen Z. Challenges and recent advances in affinity purification of tag-free proteins. Biotechnol Lett 2014; 36:1391-406.

24. Kimple ME, Brill AL, Pasker RL. Overview of affinity tags for protein purification. Curr Protoc Protein Sci 2013;73: 9.9.1-23.

25. Mauro VP, Chappell SA. A critical analysis of codon optimization in human therapeutics. Trends Mol Med 2014; 20:604-13.

26. Tanaka M, Tokuoka M, Gomi K. Effects of codon optimization on the mRNA levels of heterologous genes in filamentous fungi. Appl Microbiol Biotechnol 2014;98:385967.

27. Elena C, Ravasi P, Castelli ME, Peiru S, Menzella HG. Expression of codon optimized genes in microbial systems: current industrial applications and perspectives. Front Microbiol 2014;5:21.

28. Klement M, Liu C, Loo BL, Choo AB, Ow DS, Lee DY. Effect of linker flexibility and length on the functionality of a cytotoxic engineered antibody fragment. J Biotechnol 2015; 199:90-7.

29. Villaflores OB, Hsei CM, Teng CY, et al. Easy expression of the C-terminal heavy chain domain of botulinum neurotoxin serotype $A$ as a vaccine candidate using a bi-cistronic baculovirus system. J Virol Methods 2013;189:58-64. 